\title{
PERSPECTIVES ON ANTIVIRAL DRUGS DEVELOPMENT IN THE TREATMENT OF COVID-19
}

\author{
Aura RUSU ${ }^{1 \dagger}$, Eliza-Mihaela ARBĂNAȘI ${ }^{1 \dagger}$, Ioana-Andreea LUNGU²*, Octavia-Laura \\ MOLDOVAN $^{3}$ \\ ${ }^{1}$ Department F2, Discipline of Pharmaceutical and Therapeutical Chemistry, "George Emil Palade" \\ University of Medicine, Pharmacy, Science, and Technology of Târgu Mureș \\ ${ }^{2}$ Department F2, Discipline of Pharmacology and Clinical Pharmacy, "George Emil Palade" University of \\ Medicine, Pharmacy, Science, and Technology of Târgu Mureș \\ ${ }^{3}$ Department F1, Discipline of Organic Chemistry, "George Emil Palade" University of Medicine, Pharmacy, \\ Science, and Technology of Târgu Mureș
}

*Correspondence:

Ioana-Andreea LUNGU

ioana-andreea.lungu@umfst.ro

$\dagger$ These authors share the first authorship.

Received: 1 June 2021; Accepted: 11 June 2021; Published: 30 June 2021

\begin{abstract}
The main objective of this review is to highlight the urgent development of new antiviral drugs against SARS-CoV-2 in the context of the coronavirus pandemic. Antiviral medication against SARS-CoV-2 comprises only remdesivir as an approved drug. Scientists are making considerable efforts to identify other effective antivirals. Investments into the de novo design of new drugs against the SARS-CoV-2 virus are few. Molnupiravir proved to be effective against the SARS-CoV-2 virus and is very close to approval. Pfizer's two new compounds (PF-07321332, oral administration and PF-07304814, systemic administration) are in the early stages of development. Two types of methods are preferred to discover new antivirals in a short period. Repositioning of approved drugs for antiviral effect conducted to some clinical results for favipiravir, lopinavir/ritonavir, danoprevir/ritonavir, umifenovir, hydroxychloroquine, camostat and nafamostat. Virtual screening of known molecules' libraries indicated several compounds that were tested or are being tested in clinical trials. In conclusion, only a few innovative antiviral molecules are in various stages of development. However, the repositioning of many known compounds is being studied, including using virtual screening. The pharmaceutical industry is adapting and reinventing itself so that humanity can face a new pandemic in the future.
\end{abstract}

Keywords: COVID-19, antivirals, remdesivir, molnupiravir, hydroxychloroquine, azithromycin, ivermectin, camostat.

\section{Introduction}

Although most patients with COVID-19 have a mild or moderate course, up to $5-10 \%$ may have a severe, life-threatening course, and there is an urgent need for effective medication. Thus, more than 300 clinical trials are ongoing (Şimşek Yavuz and Ünal, 2020). Among the best known is the „Solidarity” clinical trial for
COVID-19 treatments to further evaluate remdesivir, hydroxychloroquine/chloroquine and lopinavir-ritonavir with and without interferon beta, launched by World Health Organization (WHO) (Şimşek Yavuz and Ünal, 2020; "Solidarity clinical trial for COVID-19 treatments," n.d.). 
Unfortunately, there is only one antiviral approved for treatment, namely remdesivir. Remdesivir is an antiviral that has been developed and produced by Gilead Sciences \& U.S. Centers for Disease Control Prevention (CDC) \& U.S. Army Medical Research Institute of Infectious Diseases (USAMRIID) (Eastman et al., 2020). This drug was initially used in clinical trials for the treatment of Ebola virus and Marburg virus infections (Şimşek Yavuz and Ünal, 2020), SARS-CoV, and MERS-CoV (Kouznetsov, 2020). Remdesivir has been approved as an emergency measure by the U.S. Food and Drug Administration (FDA) on 1 May 2020 to treat severe cases of COVID-19 (FDA, 2020). This antiviral molecule is an adenosine nucleotide analogue, a prodrug; the mechanism of action consists of the inhibition of viral RNA-dependent RNApolymerase $(\mathrm{RdRp})$. It is administered on the first day, $200 \mathrm{mg}$ intravenously, and then on days 2 to 5 (or to 10), only $100 \mathrm{mg}$ per day (Şimşek Yavuz and Ünal, 2020). Based on clinical trials, remdesivir was considered beneficial in treating COVID-19 due to a shorter recovery time in hospitalized adults, mainly by reducing the percentage of patients receiving invasive mechanical ventilation or Extracorporeal Membrane Oxygenation Therapy (ECMO) (Kaka et al., 2021). Treatment for a short duration of 5 days may be sufficient to treat patients with moderate or severe COVID-19 (Lai et al., 2021).

Five large randomized trials highlighted that remdesivir has many disadvantages due to a lack of decreasing the mortality rate of COVID-19 and the fact that it needs administration in a hospital. Remdesivir was more effective when it was administrated at the disease's first symptoms. Thus, the clinical efficacy of the 5-day remdesivir regimen was not assessed in critical COVID-19 patients who received mechanical ventilation or ECMO; time to recovery was not improved for those cases (Srinivas et al., 2020; Kaka et al., 2021). Therefore, the Surviving Sepsis Campaign Guidelines (SSCG) only suggest using remdesivir in non-ventilated patients with moderate to severe COVID-19. Also, SSCG are against using remdesivir in patients with critical COVID-19 outside the clinical trials (Lai et al., 2021; "SCCM | COVID-19 Guidelines," 2021).

Remdesivir treatment also involves side effects. The most common side effects of remdesivir are constipation, hypoalbuminemia, hypokalemia, anemia, thrombocytopenia and increased total bilirubin (Benlloch et al., 2020; Marcolino et al., 2020). The use of remdesivir for five days or until hospitalization is the only recommendation for patients who do not require oxygen therapy. In adult patients, remdesivir did not reduce or reduced the mortality very little, but improved the percentage of recovered patients, reduced severe damages and may lead to a decreased number of patients who become ventilated (“Antiviral Therapy," 2021; Kaka et al., 2021). However, the results of the WHO "Solidarity" study on the efficacy of remdesivir were discouraging. No drug included in the study brought significant benefits to patients hospitalized with COVID-19 ("Repurposed Antiviral Drugs for Covid-19 — Interim WHO Solidarity Trial Results," 2021). Another recent trial conducted for three months concluded, as well, that using remdesivir brought an improvement in the rehabilitation of patients with COVID-19, but that is the only advantage that the drug has had over a placebo. Even more, being costly, difficult to fabricate, and designated for intravenous use, only in hospitals, remdesivir is a less acceptable alternative (Dolgin, 2021).

The Institute for Clinical and Economic Review has updated its assessment on pricing models for remdesivir and concluded that remdesivir does not meet a critical cost- 
effectiveness threshold for a 5-day treatment course. Still, a dry powder formulation for inhalation of remdesivir is investigated. The purpose of this formulation is to increase the absorption of the drug into the lungs (Glaus MJ and Von Ruden S, 2020; Sahakijpijarn et al., 2020; Kaka et al., 2021). The new formulation of remdesivir is a promising alternative for COVID-19 treatment in preclinical studies conducted on Syrian hamsters (Sahakijpijarn et al., 2021).

An unprecedented global effort has been made to obtain the vaccines needed to achieve herd immunity (Adamson et al., 2021). Although several effective anti-COVID-19 vaccines have been approved (FDA, 2021; Pinho, 2021), the exact period of immunity for vaccinated people is yet unknown. A part of the population cannot be vaccinated, for various reasons. An essential alternative to the vaccine is the development of new efficient antivirals. However, investment in the pharmaceutical industry depends mainly on the success of approved vaccines in immunizing the population. So far, significant investments have been channeled into discovering antivirals against persistent or latent viruses, e.g., herpes (Herpes simplex), viral hepatitis, Acquired Immunodeficiency Syndrome (AIDS). The approved antiviral drugs are available for only ten viruses, although over 220 viruses are known to infect humans. COVID-19 has shown humanity that it is not prepared for a pandemic of this magnitude (Adamson et al., 2021). The antivirals discovery projects that emerged for persistent or latent viruses have enabled expertise, logistics and technology to identify new molecules efficient against SARS-CoV-2 (Richman, 2020).

It seems that the pharmaceutical industry has had an awakening after the COVID-19 pandemic. Initiatives are already in place to develop therapies against SARS-CoV-2. A program was initiated by the U.S. National
Institutes of Health (NIH) to develop treatments for viral pandemics. The pharmaceutical industry is teaming up to create new compounds to fight influenza viruses and coronaviruses. Molnupiravir is a new antiviral, a cost-effective drug, which is very close to approval in an advanced clinical trial stage (Dolgin, 2021). There are research centers such as Antiviral Drug Discovery and Development Center (of the University of Alabama at Birmingham) that aim to develop effective antiviral drugs in the treatment of COVID-19 (Everts, n.d.). The approval of a new antiviral for the treatment of COVID-19 must meet the so-called democratic conditions. The drug must be cheap, available in pharmacies for a large part of the population, without significant side effects and with some effectiveness (Benlloch et al., 2020).

The main objective of this review is to highlight the need to develop new antiviral drugs and find the most effective molecules in the treatment of COVID-19 in the shortest possible time, in the context of the SARS-CoV2 pandemic, but also of the future pandemics.

\section{The research methodology and literature review}

This review is based on relevant articles obtained by using Clarivate Analytics Web of Science and PubMed databases. The selected papers are mainly published between the last two years.

The search methodology used the main keywords and MeSH terms:

"antivirals", "antiviral agents", "COVID19", "COVID-19 treatment", "SARS-CoV-2", and "drug repurposing". The following key words were used in addition separately: "remdesivir", "favipiravir", "lopinavir", "danoprevir", "molnupiravir", "umifenovir", "hydroxychloroquine", "azithromycin", "ivermectin", and "camostat". 


\section{Methods of discovery of antiviral drugs against SARS-CoV-2 infection}

New drug development methods are timeconsuming and require significant investments (funds, logistics, and specialized human resources). Few antiviral drugs are in development, as de novo compounds (new chemical entities). To obtain more effective compounds against SARS-CoV-2 faster, repositioning of known drugs and virtual screening are preferred (Fig. 1).

\subsection{De novo design of antivirals}

Molnupiravir is a promising antiviral, prodrug of ribonucleoside analogue B-d-N4hydroxycytidine (EIDD-1931). It is an orallybioavailable form developed at Drug Innovations at Emory and licensed by Ridgeback; all funds for the development of molnupiravir have been provided by Wayne and Wendy Holman and Merck (Painter et al., 2021; Reina, 2021; Ridgeback Biotherapeutics, LP, 2021). This new antiviral is in advanced clinical study, in five phase III studies against COVID-19 (Kouznetsov, 2020; Ridgeback Biotherapeutics, LP, 2021). Molnupiravir is easy to synthesize in just three steps with a yield of $69 \%$. This method is environmentally friendly (Halford, 2021).

A new helpful oral antiviral for SARSCoV-2 has been developed by the Pfizer company from scratch, which acts by inhibiting the infection that causes COVID-19. The new molecule PF-07321332 has shown its potency in vitro by inhibiting the viral replication activity of SARS-CoV-2 (inhibitor of SARSCoV-2's main protease). This new antiviral acts as a reversible covalent inhibitor of the main protease of SARS-CoV-2 through cysteine residues. PF-07321332 is considered a potential treatment for the upcoming coronavirus risks. This oral antiviral drug would be given at the first symptoms, and patients would not need to be hospitalized. The drug is in Phase 1 clinical trial. In addition, the
Pfizer company reported PF-07304814, another compound with intravenous administration for the already hospitalized victims. The development of PF-07304814 began during the severe acute respiratory syndrome (SARS) pandemic severe acute respiratory syndrome in 2002-2003. The drug is currently in Phase $1 \mathrm{~b}$ multi-dose trial. So, having both would construct a full therapy model. The first trial will show the tolerability and pharmacokinetics of this new oral antiviral ("Pfizer Initiates Phase 1 Study of Novel Oral Antiviral Therapeutic Agent Against SARS-CoV-2 | pfpfizeruscom," 2021; "Pfizer unveils its oral SARS-CoV-2 inhibitor," 2021).

\subsection{Repositioning of known drugs}

The repositioning of a drug is the strategy to find a new biological effect other than that for which the drug was initially approved. It has many advantages, including the simplification of authorization procedures and the speed of being placed on the market at much lower costs (Jourdan et al., 2020). The most affordable alternative method for discovering the treatment of COVID-19 is the repositioning of existing drugs that are potentially active against SARS-CoV-2 (Akilesh et al., 2021; Bhowmick et al., 2021; Gatti and De Ponti, 2021).

These drugs are supposed to work upon different elements in the disease pathophysiology, and these correspond to two primary therapeutic classifications: drugs that inhibit the viral activity (e.g., favipiravir, remdesivir, azithromycin, lopinavir-ritonavir, hydroxychloroquine) and drugs that modulate the antiviral immune reaction in the host (e.g., corticosteroids, interferons, tocilizumab). Along with these, other agents have been investigated in the management of COVID-19: ivermectin - an antiparasitic agent, and doxycycline - a broad-spectrum tetracycline antibiotic (Bhowmick et al., 2021). 


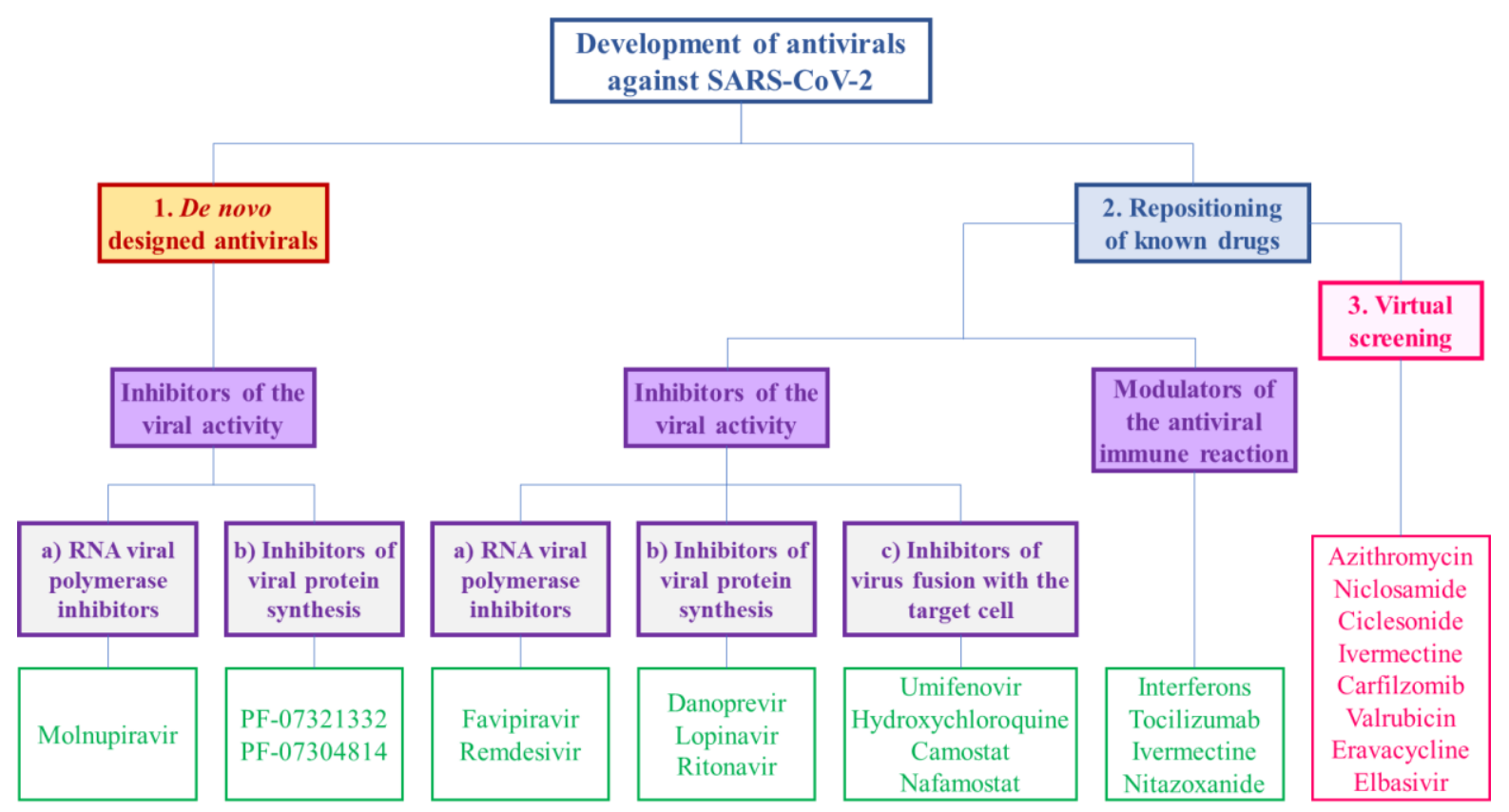

Fig. 1. Strategies for the development of antiviral drugs against SARS-CoV-2 and their classification by mechanism of action.

In a trial reported by the ISRCTN Registry and ClinicalTrials.gov, WHO specialists used four known antiviral drugs that could potentially affect mortality for patients with COVID-19. These four were: lopinavir, hydroxychloroquine, interferon (all three were discontinued), and remdesivir. The regimens used tablets of $200 \mathrm{mg}$ of lopinavir twice a day for 14 days and tablets of $200 \mathrm{mg}$ of hydroxychloroquine sulfate for ten days. Interferon beta-1 was used subcutaneously for six days with three doses of $44 \mu \mathrm{g}$ each day, and remdesivir was used intravenously with a $200 \mathrm{mg}$ dose on the first day and then just 100 $\mathrm{mg}$ for the next nine days. The study does not use any placebo. The study was conducted to test the hospital mortality for the assigned cases, and it concluded that the trial drug versus the control one had no particular benefit. Even more, none of the drugs reduced the mortality nor the hospitalization time or mechanical ventilation requirement. The similitude of all four drugs' insufficient impact proves that none has any solid effect on curing the COVID-19 infected patients. Only an individual aspect came out, that the recovery time is faster in remdesivir, compared to the other three drugs ("Repurposed Antiviral Drugs for Covid-19 - Interim WHO Solidarity Trial Results," 2021).

Supposedly, the most effective solution for COVID-19 would be an antiviral that has a component that can lower the increase of the viral load. A planned three-phase study is ongoing and focused on drugs that combat other viral diseases (e.g., daclatasvir used for hepatitis $\mathrm{C}$ virus and atazanavir used for human immunodeficiency virus (HIV)). This trial will show the changes in the increase of the viral load and measure the number of days without ventilatory support (Hospital do Coracao, 2020). A clinical trial using a combination of lopinavir-ritonavir, interferon beta- $1 \mathrm{~b}$, and ribavirin suggested an increased rate of viral clearance. Still, the conclusion was difficult to outline because there was no placebo group to compare it to, and more investigations need to be done. The central aspect that came out was that ribavirin had significant side effects and could prove dangerous to use (Lee et al., 2020). 
Also, new pharmaceutical formulations containing known drugs (e.g., remdesivir, hydroxychloroquine, heparin, pirfenidone) have been tested. The novel formulations comprise aerosol/nebulized inhalatory formulations, controlled-released formulations, nanoparticles/lipidic carriers, chiral switch. The antiviral effect was observed, alongside with the pharmacokinetic and pharmacologic properties and the safety profile (Gatti and De Ponti, 2021).

\subsection{Virtual screening in silico}

Some antivirals have been found using platforms to test the libraries with approved drugs for the in vitro action against SARSCoV-2 in cell cultures. These platforms simultaneously screen the compounds' toxicity on those cells. Thus, through this type of screening, it was discovered that azithromycin (a new generation antibiotic in the macrolide class) offers a therapeutic window in the treatment of COVID-19 (Benlloch et al., 2020). Azithromycin has been established to have antiviral and immunomodulatory effects, which may be effective in the hyperinflammatory syndrome caused by SARS-CoV-2. Azithromycin has also been clinically effective in respiratory distress syndrome and viral infections. Immunomodulatory activity is present during both the acute and the chronic inflammation phases (Pani et al., 2020; Echeverría-Esnal et al., 2021). Also, niclosamide (an anthelmintic drug) and ciclesonide (a corticosteroid drug) were selected as antivirals against SARS-CoV-2 through computational methods (Benlloch et al., 2020; Xu et al., 2020; Pérez-Moraga et al., 2021; Salvi, 2021).

A virtual screening approach against viral proteins is another method to discover a new antiviral molecule. There are in silico studies that highlight the superior efficacy of ivermectin against SARS-CoV-2 versus remdesivir and hydroxychloroquine. The affinity of ivermectin for different targets has been demonstrated (Azam et al., 2020; Choudhury et al., 2021; Kaur et al., 2021).

Ivermectin (an antiparasitic drug) was identified to interact with viral protein targets using computational methods (Kaur et al., 2021). Thus, it was shown in a clinical study that ivermectin reduced COVID-19 mortality by $40 \%$ (however, the study had some limitations) (Benlloch et al., 2020). Several drugs were shown to inhibit the primary protease in SARS-CoV-2 infection in a computational drug repositioning study. Among them are carfilzomib (an approved anticancer drug that acts as a protease inhibitor), valrubicin (a compound used in cancer chemotherapy), eravacycline (a new antibiotic by the tetracycline class used to treat intra-abdominal infections), elbasivir (an antiviral used to treat chronic hepatitis C). In vitro and in vivo studies are needed in the future to confirm the effectiveness of these drugs (Frediansyah et al., 2020; Wang, 2020).

In silico studies highlight ivermectin's efficacy versus remdesivir. The used computational methods were molecular docking and molecular dynamic simulation. Ivermectin was found to be a blocker of viral protease, replicase, and human TMPRSS2. Also, ivermectin has a sturdy hydrophobic interlinkage to the structure of the viral protease of SARS-CoV-2, and it binds better with the proteins of interest than remdesivir. The great lipophilicity and water-solubility of ivermectin lower the diffusion into the skin. With that, ivermectin is way better than remdesivir when it comes to the interaction with the ruling protease and the viral spike proteins. Even more, the pharmacokinetic characteristics of ivermectin make it a generally safe drug when used against SARSCoV-2 (Choudhury et al., 2021).

In a study conducted in silico fifteen potential COVID-19 targets were used to 
underscore ivermectin affinity. The used computational techniques were molecular docking, molecular mechanics, generalized Born surface area analysis, and molecular dynamics simulation studies. The resulted intermolecular interaction profile of ivermectin could complete the experimental studies and designing of new anti-COVID-19 drugs successfully (Azam et al., 2020).

A COVID-19 drug repurposing strategy based on virtual screening was published recently. Molecular docking and transcriptomic analyses were used to discover known drugs targeting viral proteins. A topological data analysis (TDA) compared numerous protein structures. The results obtained included 16 candidates from different pharmacological classes (Pérez-Moraga et al., 2021). In a recent docking-based virtual screening observation, using SARS-CoV-2 $\mathrm{M}^{\text {pro }}$ enzyme chymotrypsin-like cysteine protease as a target, 15 compounds from an in-house database were selected and two of them confirmed to have an in vitro ability to inhibit this protease activity. Presently, computation for these molecules is needed (Amendola et al., 2021).

\section{Classification of antiviral drugs currently undergoing studies}

The classification of the antiviral drugs currently undergoing studies (in vitro studies and clinical trials) (Şimşek Yavuz and Ünal, 2020) comprises:

- RNA viral polymerase inhibitors (inhibition of RNA synthesis)

- Inhibitors of viral protein synthesis (viral protease inhibitors, inhibitors that prevent the maturation of HIV)

- Inhibitors of virus fusion with the target cell (inhibition of viral entry)

- Immunomodulatory agents

The studied molecules with the potential to become efficient antivirals against SARS-CoV-
2 are presented below, grouped by their mechanism of action (Table 1.).

\subsection{RNA viral polymerase inhibitors}

Favipiravir is a nucleotide guanosine analogue (prodrug) (Şimşek Yavuz and Ünal, 2020) with activity against a wide variety of RNA viruses (Jomah et al., 2020; Kouznetsov, 2020). This antiviral was first approved in Japan for the treatment of influenza infections in 2014 (Agrawal et al., 2020). Favipiravir acts as an RdRp inhibitor (Şimşek Yavuz and Ünal, 2020). This simple molecule demonstrated a better efficacity compared to the lopinavirritonavir combination (Şimşek Yavuz and Ünal, 2020), (viral protease inhibitors) used to treat HIV infection (Pani et al., 2020). Also, compared to Umifenovir (Arbidol), an antiviral currently under study, favipiravir significantly improved the symptoms of pyrexia and cough, and the side effects were mild and controllable (Marcolino et al., 2020). The drug is administered orally, $1600 \mathrm{mg}$ (or $2200 \mathrm{mg}$ ) on the first day, twice a day (Jomah et al., 2020), and on days 2 to 7 (or 10), $600 \mathrm{mg}$ are given twice a day (Jomah et al., 2020; Şimşek Yavuz and Ünal, 2020).

Overall, favipiravir was well tolerated (in all five completed clinical trials) (Jomah et al., 2020). Adverse reactions such as diarrhea, psychiatric reactions, liver toxicity, hyperuricemia have been reported, but most of them have disappeared until patients were discharged. The safety of favipiravir is currently being investigated (Agrawal et al., 2020; Jomah et al., 2020; Marcolino et al., 2020; Dabbous et al., 2021). Favipiravir presents some valuable advantages, like oral administration and the recommendation to nonhospitalized patients with mild to moderate condition. This antiviral drug could be used relatively safely in the treatment of a large number of patients with COVID-19 (Agrawal et al., 2020). 
Table 1. Chemical structures of antivirals with potential against SARS-CoV-2.

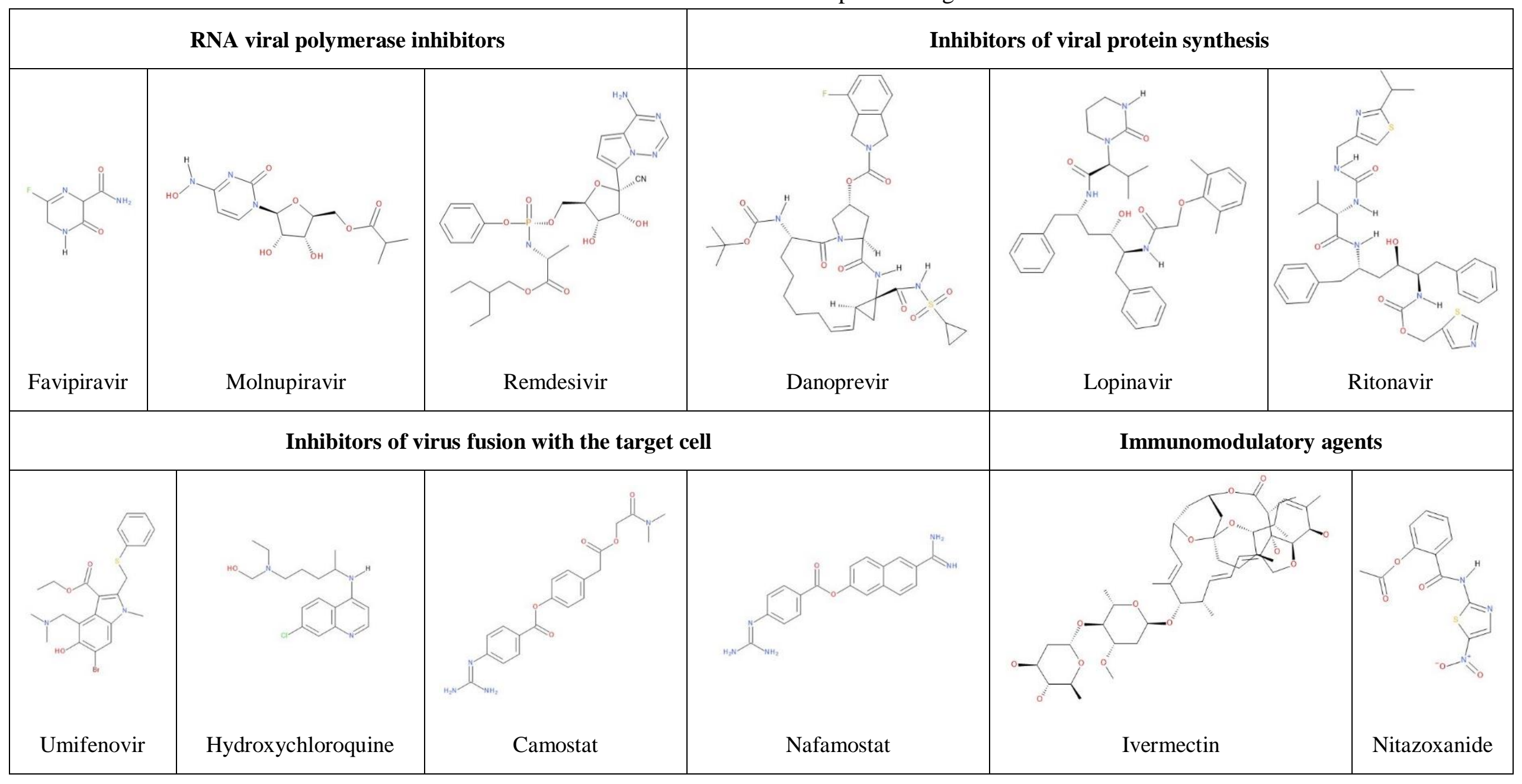


Molnupiravir (EIDD-2801) is an antiviral, prodrug of ribonucleoside analogue Bd-N4-hydroxycytidine. This compound has been proven to be active against numerous RNA viruses (Painter et al., 2021; Reina, 2021). The active form blocks RNA polymerase, an essential component of viral replication. Molnupiravir also acts by RNA mutagenesis via the template strand (Gordon et al., 2021). This potential anti-COVID-19 drug is administrated orally. The dosing of this drug is from $50 \mathrm{mg}$ to up to $1600 \mathrm{mg}$ (as a single dose). Still, administering it has proved to be effective to treat pathogenic respiratory RNA viruses and improving the function of the lungs by just giving lower doses of a maximum of $500 \mathrm{mg}$. Its side effects are relatively low, primarily headaches and diarrhea; when the dose is higher than $800 \mathrm{mg}$, the substance has to be formulated into an oral solution (Painter et al., 2021). Compared to remdesivir, molnupiravir is given orally and is generally well tolerated (Painter et al., 2021). Therefore, it has a high chance of being approved and administered in the treatment of COVID-19, being in advanced clinical study, in five phase III studies against COVID-19 (Kouznetsov, 2020; Ridgeback Biotherapeutics, LP, 2021).

\subsection{Inhibitors of viral protein synthesis}

There are antivirals in this group that have been shown to have some potential in the treatment of COVID-19.

Lopinavir/ritonavir is a combination of two inhibitors of viral protein synthesis used in the treatment of HIV infection (Jomah et al., 2020; Kouznetsov, 2020; Şimşek Yavuz and Ünal, 2020) but with limited efficacy against SARS-CoV-2 (Teoh et al., 2020). The combination is administered orally twice a day, in dosages of $400 \mathrm{mg} / 100 \mathrm{mg}$ (1-10 or 14 days) (Şimşek Yavuz and Ünal, 2020). It is recommended to avoid higher doses due to severe gastrointestinal side effects (loss of appetite, diarrhea, nausea, vomiting), hypokalemia, self-limiting rash and increased level of alanyl transferase.

Many side effects and drug interactions occur with lopinavir/ritonavir as a result of strong inhibition of CYP3A4 (Jomah et al., 2020; Teoh et al., 2020; Srinivas et al., 2020). The occurrence of these severe side effects has contributed to the discontinuation of therapy in some studies (Jomah et al., 2020; Teoh et al., 2020).

The WHO tried to reposition lopinavir through the international "Solidarity" clinical trial, but without success. The results indicated lesser effects or lack of effects in COVID-10 treatment ("Repurposed Antiviral Drugs for Covid-19 - Interim WHO Solidarity Trial Results," 2021). An essential disadvantage of lopinavir/ritonavir is the occurrence of drug interactions (Teoh et al., 2020). Lopinavir/ritonavir is not included in the recommendations of the US National Institutes of Health (NIH) guidelines for the treatment of COVID-19, except for a clinical study (“Antiviral Therapy," 2021).

Danoprevir/ritonavir is another combination that can be used in the treatment of COVID-19. Danoprevir is known to be a potent hepatitis $\mathrm{C}$ virus (HCV) protease inhibitor. Since 2018, it has been approved and marketed in China as a direct-acting antiviral agent (oral administration), potentiated by another viral protease inhibitor, ritonavir, and interferon. Following the therapeutical success of three patients infected with SARS-CoV-2, the hypothesis that this combination may be effective for patients with a moderate form of COVID-19 was issued (Marcolino et al., 2020). Another recent clinical trial enrolled 11 patients who tolerated the combination of danoprevir and ritonavir well, without composite side effects (Chen et al., 2020). In a study conducted on 33 COVID-19 patients danoprevir has been proven to be an appropriate treatment plan due to a shorter 
hospitalization period compared to lopinavir/ritonavir (Zhang et al., 2020).

\subsection{Inhibitors of virus fusion with the}

\section{target cell}

Umifenovir is an indole derivative (approved in China and Russia for the treatment of influenza A and B virus) with activity against both encapsulated and capsulefree viruses. In vitro, Umifenovir has effective antiviral activity against SARS-Cov-2, but is still undergoing study (Frediansyah et al., 2020; Jomah et al., 2020). Its primary therapeutic action is still uncertain. It is not clear if it has a direct antiviral activity, if it stimulates the immune system or has antiinflammatory action (Kouznetsov, 2020). The main advantage of Umifenovir is the lack of significant side effects, with only mild gastrointestinal side effects reported in some patients (Jomah et al., 2020).

\section{Hydroxychloroquine and chloroquine} belong to this category of antivirals. The effects of the two compounds (usually used as antimalarials or to treat autoimmune diseases) are: reduction of pneumonic symptoms, a significant reduction in viral load and a shorter average duration of treatment (Marcolino et al., 2020). The mechanisms of action are multiple, related to viral replication, endosomal $\mathrm{pH}$, glycosylation process, modification of viral proteins, and activity of the immune system (Marcolino et al., 2020; Şimşek Yavuz and Ünal, 2020). The oral administration of drugs has the following treatment schedule: $200 \mathrm{mg}$ twice a day, during 1-5 days for hydroxychloroquine and $500 \mathrm{mg}$ twice a day, during 1-5 (or 10) days for chloroquine (Şimşek Yavuz and Ünal, 2020). According to the World Health Organization (WHO), the efficacy and safety of hydroxychloroquine for the treatment of COVID-19 are debatable ("Targeted Update," 2020). In combination with azithromycin (an antibiotic from a new generation of macrolides), hydroxychloroquine was significantly more effective (Marcolino et al., 2020). Azithromycin is known to have immunomodulatory and antiviral properties (Pani et al., 2020).

However, the recommendations of the NIH guideline do not include the use of hydroxychloroquine with or without azithromycin for both hospitalized and nonhospitalized patients diagnosed with COVID19. Also, the NIH guide does not recommend using high doses of chloroquine for the treatment of COVID-19 ("Antiviral Therapy," 2021). Unfortunately, hydroxychloroquine was also found inefficient when used in the regimen of the hospitalized COVID-19 patients by the results of the "Solidarity" clinical trial ("Repurposed Antiviral Drugs for Covid-19 Interim WHO Solidarity Trial Results," 2021).

Camostat (mesylate) is a serine protease inhibitor (a synthetic proteolytic enzyme inhibitor for trypsin, plasmin, kallikrein, tissue kallikrein and thrombin) (Frediansyah et al., 2020; Marcolino et al., 2020). This drug has been shown to be effective in treating COVID19 , reducing mortality, with a survival rate of $60 \%$. The proper dosage of the compound to control viral spread is not yet known (Marcolino et al., 2020).

Nafamostat (mesylate) is also a serine protease inhibitor approved in Japan to treat acute pancreatitis. Currently, it is being studied for COVID-19 treatment (Frediansyah et al., 2020; Marcolino et al., 2020).

\subsection{Immunomodulatory agents}

Ivermectin is an antiparasitic agent approved by the FDA that has shown action for the RNA and DNA viruses. Thus, ivermectin has been studied for its antiviral activity against a wide range of viruses in vitro. It inhibits HIV replication and limits retrovirus, adenovirus and pseudorabies virus (PRV) infection both in vitro and in vivo. However, no efficacy of this drug against the Zika virus has been observed. Therefore, ivermectin has been promising for 
treating COVID-19 in in vitro studies, even proving its effect on inhibiting the replication of the SARS-CoV-2 virus (Marcolino et al., 2020; Bello, 2021). Its binding interaction mediates the potential therapeutic mechanism to the target sites such as importin $\alpha / \beta$ (IMP $\alpha / \beta)$-mediated nuclear transport of HIV-1 integrase, NS5 polymerase, NS3 helicase, nuclear import of UL42, and nuclear localization signal mediated nuclear import of Cap (Caly et al., 2020; Şimşek Yavuz and Ünal, 2020; Taher et al., 2021). However, the $\mathrm{NIH}$ guide does not recommend either for or against the use of ivermectin for the treatment of COVID-19, except for a clinical study (“Antiviral Therapy," 2021).

Nitazoxanide is an antiparasitic drug that has been clinically authorized and commercialized for its antiviral activity. It works by interfering with the route of the type 1 interferons and viral duplication. It has been demonstrated that it represses the replication of many viruses, such as influenza viruses, MERS, hepatitis B and C and other pulmonary viruses (Eastman et al., 2020). Furthermore, it has been shown to inhibit SARS-CoV-2 replication at low micromolar concentrations in Vero CCL81 cells (Kouznetsov, 2020). In addition, nitazoxanide is orally bioavailable and broadly well-tolerated, thus representing a promising alternative for the management of COVID-19 were it to prove effective in vivo. In a recent study, patients with mild Covid-19 symptoms and who were given nitazoxanide (500 $\mathrm{mg}$ ) for five days showed no advantage and no symptom reduction versus the placebo groups. Early nitazoxanide therapy was safe and reduced viral load significantly, and no serious adverse events were observed (Rocco et al., 2020). Even increasing the dose had no adverse events, most patients just reported feeling nauseous, having headaches or diarrhea. The primary outcome is that nitazoxanide reduced the viral load, compared to the placebo, if given at the early symptoms of SARS-CoV-2 (Mendieta Zerón et al., 2021).

Many other drugs may be repositioned as effective medicines (as immunomodulators) in the treatment of COVID-19, such as tocilizumab, ribavirin, ruxolitinib, ingavirin etc. (Kouznetsov, 2020). Antivirals have little effect on mortality in patients hospitalized with COVID-19, suggest WHO interim trial results (Robinson, 2020). A triple combination of interferon beta-1b with lopinavir-ritonavir and ribavirin was also tested in a clinical study (Hung et al., 2020). The clinical efficacy of this triple combination is challenging to assess due to the absence of a more appropriate control group and the questionable effectiveness of lopinavir-ritonavir and ribavirin against SARSCov-2, respectively. However, the results showed accelerated viral clearance in these combinations with interferon beta- $1 \mathrm{~b}$ (Hung et al., 2020; Lee et al., 2020).

\section{Conclusions}

The COVID-19 pandemic has restarted the pharmaceutical industry in terms of antiviral therapy. The initial lack of an effective SARSCoV-2 vaccine has led to numerous studies and trials of many existing drugs, from antiviral and other drug classes. Although several vaccines have been authorized internationally, the need for effective antivirals against SARSCoV-2 has remained pressing. The range of antivirals available in the pharmaceutical market has been addressed mainly for persistent or latent viruses, such as HIV or hepatitis viruses. Significant investments for the development of new antivirals have been missing in SARS-type viruses, the focus being mainly on producing vaccines. A global effort is needed to develop new effective antiviral drugs, in order to prevent another global pandemic situation. By analyzing the most recently published data, we identified three 
strategies for developing new antiviral compounds, useful in the treatment of COVID19 and other coronavirus pandemics. The first strategy, de novo design, is time and resources consuming. Therefore, many resources are channeled towards faster development strategies, such as repositioning already known drugs or the virtual screening of the extensive library of known compounds. The development of new drugs from scratch is less preferred. Repositioning of available drugs is a desirable alternative that will provide effective compounds in the treatment of COVID-19 in a short time. Also, virtual screening (in silico methods) is a modern alternative with many advantages, among the most important being the decreasing of costs and the shorter time for identification of lead compounds with potential in the regimens of COVID-19. More extensive randomized controlled trials are needed to identify the best candidates, including antiCOVID-19 therapeutic combinations. Based on the correlated data, we are optimistic that there is an excellent chance that new antiviral drugs effective in the treatment of COVID-19 will be approved in the near future.

\section{Conflict of interest}

The authors declare that the research was conducted in the absence of any commercial or financial relationships that could be construed as a potential conflict of interest.

\section{References}

1. Adamson CS, Chibale K, Goss RJM, Jaspars M, Newman DJ, Dorrington RA (2021) Antiviral drug discovery: preparing for the next pandemic. Chem Soc Rev 50:3647-3655. doi: 10.1039/D0CS01118E

2. Agrawal U, Raju R, Udwadia ZF (2020) Favipiravir: A new and emerging antiviral option in COVID-19. Med J Armed Forces India 76:370-376. doi: 10.1016/j.mjafi.2020.08.004

3. Akilesh SM, J R, Palanisamy D, Wadhwani A (2021) Repositioning of Drugs to Counter COVID-19 Pandemic - An Insight. Curr Pharm Biotechnol 22:192-199. doi:10.2174/1389201021999200820155927

4. Amendola G, Ettari R, Previti S, Di Chio C, Messere A, Di Maro S, Hammerschmidt SJ, Zimmer C, Zimmermann RA, Schirmeister T, Zappalà M, Cosconati S (2021) Lead Discovery of SARS-CoV-2 Main Protease Inhibitors through Covalent Docking-Based Virtual Screening. J Chem Inf Model 61:2062-2073.

doi: 10.1021/acs.jcim.1c00184

5. Antiviral Therapy [WWW Document] (Last updated: 11 Feb 2021) COVID-19 Treatment Guidelines.

https://www.covid19treatmentguidelines.ni h.gov/antiviral-therapy/ Accessed 20 May 2021

6. Azam F, Taban IM, Eid EEM, Iqbal M, Alam O, Khan S, Mahmood D, Anwar MJ, Khalilullah H, Khan MU (2020) An insilico analysis of ivermectin interaction with potential SARS-CoV-2 targets and host nuclear importin $\alpha$. J Biomol Struct Dyn 1-14. doi: 10.1080/07391102.2020.1841028

7. Bello M (2021) Elucidation of the inhibitory activity of ivermectin with host nuclear importin $\alpha$ and several SARS-CoV2 targets. J Biomol Struct Dyn 1-9. doi: 10.1080/07391102.2021.1911857

8. Benlloch J-M, Cortés J-C, MartínezRodríguez D, Julián R-S, Villanueva R-J (2020) Effect of the early use of antivirals on the COVID-19 pandemic. A computational network modeling approach. Chaos Soliton Fract 140:110168. doi: 10.1016/j.chaos.2020.110168

9. Bhowmick S, Dang A, Vallish BN, Dang S (2021) Safety and Efficacy of Ivermectin and Doxycycline Monotherapy and in 
Combination in the Treatment of COVID19: A Scoping Review. Drug Saf.

doi: 10.1007/s40264-021-01066-y

10. Caly L, Druce JD, Catton MG, Jans DA, Wagstaff KM (2020) The FDA-approved drug ivermectin inhibits the replication of SARS-CoV-2 in vitro. Antivir Res 178:104787.

doi: 10.1016/j.antiviral.2020.104787

11. Chen H, Zhang Z, Wang L, Huang Z, Gong F, Li X, Chen Y, Wu JJ (2020) First clinical study using HCV protease inhibitor danoprevir to treat COVID-19 patients. Medicine (Baltimore) 99.

doi: 10.1097/MD.0000000000023357

12. Choudhury A, Das NC, Patra R, Bhattacharya M, Ghosh P, Patra BC, Mukherjee S (2021) Exploring the binding efficacy of ivermectin against the key proteins of SARS-CoV-2 pathogenesis: an in silico approach. Future Virol 16:277291. doi: 10.2217/fvl-2020-0342

13. Dabbous HM, El-Sayed MH, El Assal G, Elghazaly H, Ebeid FFS, Sherief AF, Elgaafary M, Fawzy E, Hassany SM, Riad AR, TagelDin MA (2021) Safety and efficacy of favipiravir versus hydroxychloroquine in management of COVID-19: A randomised controlled trial. Sci Rep-UK 11:7282. doi: 10.1038/s41598021-85227-0

14. Dolgin E (2021) The race for antiviral drugs to beat COVID - and the next pandemic. Nature 592:340-343. doi: 10.1038/d41586-021-00958-4

15. Eastman RT, Roth JS, Brimacombe KR, Simeonov A, Shen M, Patnaik S, Hall MD (2020) Remdesivir: A Review of Its Discovery and Development Leading to Emergency Use Authorization for Treatment of COVID-19. ACS Cent Sci 6:672-683.

doi: $10.1021 /$ acscentsci.0c00489
16. Echeverría-Esnal D, Martin-Ontiyuelo C, Navarrete-Rouco ME, Cuscó MD-A, Ferrández O, Horcajada JP, Grau S (2021) Azithromycin in the treatment of COVID19: a review. Expert Rev Anti-infe 19:147163.

doi: 10.1080/14787210.2020.1813024

17. Everts M, n.d. Antiviral Drug Discovery and Development Center | UAB [WWW Document]. https://www.uab.edu/medicine/ad3c/ Accessed 20 May 2021

18. FDA (2020) Coronavirus (COVID-19) Update: FDA Issues Emergency Use Authorization for Potential COVID-19 Treatment [WWW Document]. FDA. https://www.fda.gov/news-events/pressannouncements/coronavirus-covid-19update-fda-issues-emergency-useauthorization-potential-covid-19-treatment Accessed 30 Sep 2020

19. FDA (2021) COVID-19 Vaccines. https://www.fda.gov/emergencypreparedness-and-response/coronavirusdisease-2019-covid-19/covid-19-vaccines Accessed 21 May 2021

20. Frediansyah A, Tiwari R, Sharun K, Dhama K, Harapan H (2021) Antivirals for COVID-19: A critical review. Clin Epidemiol Glob Health 9:90-98. doi: 10.1016/j.cegh.2020.07.006

21. Gatti M, De Ponti F (2021) Drug Repurposing in the COVID-19 Era: Insights from Case Studies Showing Pharmaceutical Peculiarities.

Pharmaceutics 13. doi: 10.3390/pharmaceutics13030302

22. Gordon CJ, Tchesnokov EP, Schinazi RF, Götte M (2021) Molnupiravir promotes SARS-CoV-2 mutagenesis via the RNA template. J Biol Chem. doi: 10.1016/j.jbc.2021.100770

23. Glaus MJ, Von Ruden S (2020) Remdesivir and COVID-19. The Lancet 396:952. 
doi: 10.1016/S0140-6736(20)32021-3

24. Halford B (2021) PROCESS CHEMISTRY Chemists shorten synthesis of molnupiravir. Chem. Eng. News 99, 7-7.

25. Hospital do Coracao (2020) Antiviral for Adult Patients Hospitalized for SARSCoV-2 Infection: a Randomized, Phase 2/3, Multicenter, Placebo Controlled, Adaptive, Multi-arm, Multi-stage Clinical Trial Coalition Brazil COVID-19 IX: REVOLUTIOn (Clinical trial registration No. NCT04468087). clinicaltrials.gov.

26. Hung IF-N, Lung K-C, Tso EY-K, Liu R, Chung TW-H, Chu M-Y, Ng Y-Y, Lo J, Chan J, Tam AR, Shum H-P, Chan V, Wu AK-L, Sin K-M, Leung W-S, Law W-L, Lung DC, Sin S, Yeung P, Yip CC-Y, Zhang RR, Fung AY-F, Yan EY-W, Leung K-H, Ip JD, Chu AW-H, Chan W-M, Ng AC-K, Lee R, Fung K, Yeung A, Wu T-C, Chan JW-M, Yan W-W, Chan W-M, Chan JF-W, Lie AK-W, Tsang OT-Y, Cheng VC-C, Que T-L, Lau C-S, Chan K-H, To KK-W, Yuen K-Y (2020) Triple combination of interferon beta- $1 b$, lopinavir-ritonavir, and ribavirin in the treatment of patients admitted to hospital with COVID-19: an open-label, randomised, phase 2 trial. The Lancet 395:1695-1704. doi: 10.1016/S01406736(20)31042-4

27. Jomah S, Asdaq SMB, Al-Yamani MJ (2020) Clinical efficacy of antivirals against novel coronavirus (COVID-19): A review. J Infect Public Heal 13:1187-1195. doi: 10.1016/j.jiph.2020.07.013

28. Jourdan J-P, Bureau R, Rochais C, Dallemagne P (2020) Drug repositioning: a brief overview. J Pharm Pharmacol 72:1145-1151. doi: 10.1111/jphp.13273

29. Kaka AS, MacDonald R, Greer N, Vela K, Duan-Porter W, Obley A, Wilt TJ (2021) Major Update: Remdesivir for Adults With
COVID-19: A Living Systematic Review and Meta-analysis for the American College of Physicians Practice Points. Ann Intern Med. doi: 10.7326/M20-8148

30. Kaur H, Shekhar N, Sharma S, Sarma P, Prakash A, Medhi B (2021) Ivermectin as a potential drug for treatment of COVID-19: an in-sync review with clinical and computational attributes. Pharmacol Rep. doi: 10.1007/s43440-020-00195-y

31. Kouznetsov VV (2020) COVID-19 treatment: Much research and testing, but far, few magic bullets against SARS-CoV-2 coronavirus. Eur J Med Chem 203:112647. doi: 10.1016/j.ejmech.2020.112647

32. Lai C-C, Chen C-H, Wang C-Y, Chen K-H, Wang Y-H, Hsueh P-R (2021) Clinical efficacy and safety of remdesivir in patients with COVID-19: a systematic review and network meta-analysis of randomized controlled trials. J Antimicrob Chemother. doi: 10.1093/jac/dkab093

33. Lee N, Ison M, Dunning J (2020) Early triple antiviral therapy for COVID-19. The Lancet 396:1487-1488. doi: 10.1016/S0140-6736(20)32274-1

34. Marcolino VA, Pimentel TC, Barão CE (2020) What to expect from different drugs used in the treatment of COVID-19: A study on applications and in vivo and in vitro results. Eur J Pharmacol 887:173467. doi: 10.1016/j.ejphar.2020.173467

35. Mendieta Zerón H, Meneses Calderón J, Paniagua Coria L, Meneses Figueroa J, Vargas Contreras MJ, Vives Aceves HL, Carranza Salazar FM, Californias Hernández D, Miraflores Vidaurri E, Carrillo González A, Anaya Herrera J (2021) Nitazoxanide as an early treatment to reduce the intensity of COVID-19 outbreaks among health personnel. World Academy of Sciences Journal 3:1-6. doi: 10.3892/wasj.2021.94 
36. Painter WP, Holman W, Bush JA, Almazedi F, Malik H, Eraut NCJE, Morin MJ, Szewczyk LJ, Painter GR (2021) Human Safety, Tolerability, and Pharmacokinetics of Molnupiravir, a Novel Broad-Spectrum Oral Antiviral Agent with Activity Against SARS-CoV-2. Antimicrob Agents Chemother. doi: 10.1128/AAC.02428-20

37. Pani A, Lauriola M, Romandini A, Scaglione F (2020) Macrolides and viral infections: focus on azithromycin in COVID-19 pathology. Int J Antimicrob Ag 56:106053.

doi: 10.1016/j.ijantimicag.2020.106053

38. Pérez-Moraga R, Forés-Martos J, SuayGarcía B, Duval J-L, Falcó A, Climent J (2021) A COVID-19 Drug Repurposing Strategy through Quantitative Homological Similarities Using a Topological Data Analysis-Based Framework. Pharmaceutics 13:488.

doi: 10.3390/pharmaceutics13040488

39. Pfizer Initiates Phase 1 Study of Novel Oral Antiviral Therapeutic Agent Against SARS-CoV-2 | pfpfizeruscom [WWW Document] (23 Mar 2021) https://www.pfizer.com/news/pressrelease/press-release-detail/pfizer-initiatesphase-1-study-novel-oral-antiviral Accessed 21 May 2021

40. Pfizer unveils its oral SARS-CoV-2 inhibitor [WWW Document] (07 Apr 2021) Chemical \& Engineering News. https://cen.acs.org/acs-news/acs-meetingnews/Pfizer-unveils-oral-SARSCoV/99/i13 Accessed 21 May 2021

41. Pinho AC (2021) COVID-19 vaccines [WWW Document]. European Medicines Agency. https://www.ema.europa.eu/en/humanregulatory/overview/public-healththreats/coronavirus-disease-covid- 19/treatments-vaccines/covid-19-vaccines Accessed 19 May 2021

42. Reina J (2021) [Plitidepsin, an inhibitor of the cell elongation factor eEF1a, and molnupiravir an analogue of the ribonucleoside cytidine, two new chemical compounds with intense activity against SARS-CoV-2]. Rev Esp Quimioter. doi: 10.37201/req/042.2021

43. Repurposed Antiviral Drugs for Covid-19 - Interim WHO Solidarity Trial Results (2021) New Engl J Med 384:497-511. doi: 10.1056/NEJMoa2023184

44. Richman DD (2020) Antiviral Drug Discovery To Address the COVID-19 Pandemic. mBio 11. doi: $10.1128 / \mathrm{mBio} .02134-20$

45. Ridgeback Biotherapeutics, LP (2021) The Safety of EIDD-2801 and Its Effect on Viral Shedding of SARS-CoV-2 (Clinical trial registration No. NCT04405739). clinicaltrials.gov.

46. Robinson J (19 Oct 2020) Antivirals have little effect on mortality in patients hospitalised with COVID-19, suggest WHO trial interim results [WWW Document]. The Pharmaceutical Journal. https://pharmaceuticaljournal.com/article/news/antivirals-havelittle-effect-on-mortality-in-patientshospitalised-with-covid-19-suggest-whotrial-interim-results Accessed 30 Apr 2021

47. Rocco PRM, Silva PL, Cruz FF, Junior MACM, Tierno PFGMM, Moura MA, Oliveira LFGD, Lima CC, Santos EAD, Junior WF, Fernandes APSM, Franchini KG, Magri E, Moraes NF, de Gonçalves JMJ, Carbonieri MN, Santos ISD, Paes NF, Maciel PVM, Rocha RP, Carvalho AF, de Alves PA, Modena JLP, Cordeiro AT, Trivella DBB, Marques RE, Luiz RR, Pelosi P, Silva JRL (2020) Early use of nitazoxanide in mild Covid-19 disease: randomised, placebo-controlled trial. Eur 
Respir J. doi: 10.1183/13993003.037252020

48. Sahakijpijarn S, Moon C, Koleng JJ, Christensen DJ, Williams RO (2020) Development of Remdesivir as a Dry Powder for Inhalation by Thin Film Freezing. Pharmaceutics 12:1002. doi: 10.3390/pharmaceutics12111002

49. Sahakijpijarn S, Moon C, Warnken ZN, Maier EY, DeVore JE, Christensen DJ, Koleng JJ, Williams RO (2021) In vivo pharmacokinetic study of remdesivir dry powder for inhalation in hamsters. International Journal of Pharmaceutics: $\mathrm{X}$ 3:100073.

doi: 10.1016/j.ijpx.2021.100073

50. Salvi SS (2021) Is there a role for inhaled ciclesonide in the treatment of COVID-19? Lung India 38:1-4. doi: 10.4103/lungindia.lungindia_473_20

51. SCCM | COVID-19 Guidelines [WWW Document] (29 Jan 2021) Society of Critical Care Medicine (SCCM). https://sccm.org/SurvivingSepsisCampaign/ Guidelines/COVID-19 Accessed 12 May 2021

52. Şimşek Yavuz S, Ünal S (2020) Antiviral treatment of COVID-19. Turk J Med Sci 50:611-619. doi: 10.3906/sag-2004-145

53. Solidarity clinical trial for COVID-19 treatments [WWW Document], n.d. https://www.who.int/emergencies/diseases/ novel-coronavirus-2019/global-researchon-novel-coronavirus-2019-ncov/solidarityclinical-trial-for-covid-19-treatments

Accessed 12 Dec 2020

54. Srinivas P, Sacha G, Koval C (2020) Antivirals for COVID-19. Clev Clin J Med. doi: 10.3949/ccjm.87a.ccc030

55. Taher M, Tik N, Susanti D (2021) Drugs intervention study in COVID-19 management. Drug Metab Pers Ther. doi: 10.1515/dmdi-2020-0173
56. Targeted Update: Safety and efficacy of hydroxychloroquine or chloroquine for treatment of COVID-19 [WWW Document] (17 Jun 2020) https://www.who.int/publications/m/item/ta rgeted-update-safety-and-efficacy-ofhydroxychloroquine-or-chloroquine-fortreatment-of-covid-19 Accessed 13 Oct 2020

57. Teoh SL, Lim YH, Lai NM, Lee SWH (2020) Directly Acting Antivirals for COVID-19: Where Do We Stand? Front Microbiol 11. doi: 10.3389/fmicb.2020.01857

58. Wang J (2020) Fast Identification of Possible Drug Treatment of Coronavirus Disease $\quad-19$ (COVID-19) Through Computational Drug Repurposing Study. doi: 10.26434/chemrxiv.11875446.v1

59. Xu J, Shi P-Y, Li H, Zhou J (2020) Broad Spectrum Antiviral Agent Niclosamide and Its Therapeutic Potential. ACS Infect Dis. doi: 10.1021/acsinfecdis.0c00052

60. Zhang Z, Wang S, Tu X, Peng X, Huang Y, Wang L, Ju W, Rao J, Li X, Zhu D, Sun H, Chen H (2020) A comparative study on the time to achieve negative nucleic acid testing and hospital stays between danoprevir and lopinavir/ritonavir in the treatment of patients with COVID-19. J Med Virol 92:2631-2636. doi: 10.1002/jmv.26141 